\title{
Transcriptional Regulation of the Kaposi's Sarcoma-Associated Herpesvirus K15 Gene
}

\author{
Emily L. Wong ${ }^{1}$ and Blossom Damania ${ }^{2 *}$ \\ Department of Internal Medicine, Division of Infectious Diseases, ${ }^{1}$ and Department of Microbiology and Immunology, ${ }^{2}$ \\ University of North Carolina, Chapel Hill, North Carolina 27599
}

Received 1 August 2005/Accepted 14 November 2005

\begin{abstract}
The K15 gene product of Kaposi's sarcoma-associated herpesvirus (KSHV) is a transmembrane protein that is encoded by the last open reading frame of the KSHV genome. The K15 protein has been implicated in modulation of B-cell signal transduction and activation of the Ras/mitogen-activated protein kinase and NF-кB signal transduction pathways. Here we report the identification of the transcriptional start site of the fulllength K15 gene in KSHV-positive BCBL-1 cells. We have mapped the K15 transcriptional start site to a position 152 nucleotides upstream from the translation start site by rapid amplification of cDNA ends and RNase protection assays. We have also characterized the K15 promoter element. To analyze the cis-acting elements necessary to regulate K15 gene expression, a series of 5' promoter deletion constructs were generated and subcloned upstream of the luciferase reporter gene. Transcriptional assays with these mutant promoters demonstrated that chemical induction in latently infected KSHV-positive BCBL-1 cells activated K15 transcription. In addition, K15 promoter transactivation was also mediated by the viral immediate-early protein Orf50/Rta, suggesting that the K15 gene is actively transcribed during lytic replication.
\end{abstract}

Kaposi's sarcoma-associated herpesvirus (KSHV), also known as human herpesvirus 8, was first discovered in 1994 by Chang et al. in Kaposi's sarcoma (KS) lesions procured from patients with AIDS (7). Subsequently, KSHV DNA sequences were detected in all four types of KS (1): (i) classic KS, which affects elderly men of Mediterranean and eastern European descent; (ii) endemic KS, which is highly prevalent in sub-Saharan Africa (28); (iii) iatrogenic or immunosuppressive KS, which develops in solidorgan transplant recipients $(4,26)$; and (iv) AIDS KS. In addition, $\mathrm{KSHV}$ is implicated as the etiologic agent of two lymphoproliferative disorders in both human immunodeficiency virus-positive and human immunodeficiency virus-negative individuals, body cavity-based lymphoma (BCBL) or primary effusion lymphoma (PEL) $(5,14,21)$, and the plasmablastic variant of multicentric Castleman's disease (32). PEL is a form of non-Hodgkin's lymphoma that presents as malignant effusions in pleural, pericardial, or peritoneal cavities without a detectable tumor mass or, alternatively, manifests as a solid mass in lymph nodes, lungs, or the gastrointestinal tract (35). KSHV exhibits cell tropism for vascular endothelial cells, epithelial cells, and B cells (20).

$\mathrm{KSHV}$ is classified as a member of the herpesvirus family, a group of enveloped viruses containing double-stranded DNA (1). KSHV is a member of the gamma subgroup of herpesviruses, which is further subdivided into two genera: lymphocryptoviruses (gamma-1) and rhadinoviruses (gamma-2). Members of the gamma-1 herpesvirus subfamily include Epstein-Barr virus, Lymphocryptovirus of rhesus monkeys, and Herpesvirus papio of baboons. The gamma- 2 herpesvirus subfamily is comprised of KSHV, Herpesvirus saimiri, Rhesus monkey rhadinovirus, and Murine herpesvirus 68 (9). There are 81 open reading frames

\footnotetext{
* Corresponding author. Mailing address: Lineberger Comprehensive Cancer Center, CB 7295, University of North Carolina, Chapel Hill, NC 27599. Phone: (919) 843-6011. Fax: (919) 966-9673. E-mail: damania@med.unc.edu.
}

(ORFs) in the long unique region of the KSHV genome flanked by terminal repeats $(24)$.

Like other herpesviruses, KSHV undergoes temporal and coordinated expression of three kinetic classes of genes during lytic expression. These are the immediate-early, early, and late genes (34). The KSHV immediate-early gene product, Orf50/ $\mathrm{Rta}$, is a potent transactivator with a central role in regulating the switch from viral latency to lytic replication and is sufficient to activate the cascade of viral replication resulting in the production of encapsidated virions $(18,33,37,39)$.

The KSHV K15 transcript has been detected in latently infected cells; however, transcript levels increase following chemical induction to lytic infection $(8,13,23,27)$. The K15 gene is encoded by the last ORF of the KSHV genome, adjacent to the terminal repeats, and is transcribed in a leftward orientation away from the terminal repeat region $(8,13,23)$. The K15 gene contains eight exons which undergo alternative splicing, yielding several isoforms with a predicted common carboxy-terminal cytoplasmic tail and different numbers of transmembrane domains $(8,13,23)$. The longest $\mathrm{K} 15$ isoform contains 12 membrane-spanning domains. Analysis of KSHV strains from different regions of the world suggests that K15 exists as two highly diverged allelic forms, the 2,500-bp $\mathrm{P}$ form ( $\mathrm{P}$ for predominant) and the 3,500-bp $\mathrm{M}$ allele ( $\mathrm{M}$ for minor) $(8,13,23,38)$. Although the $\mathrm{P}$ and $\mathrm{M}$ forms display less than $30 \%$ amino acid homology to one another (23), the predicted protein structure is highly conserved between the two.

Similar to Epstein-Barr virus LMP2A, K15 is able to block B-cell receptor signal transduction (8). The cytoplasmic tail of K15 contains conserved SH2 binding motifs which are known to interact with the Src and Syk families of protein tyrosine kinases (8). The Src protein tyrosine kinase family members, Src, Fyn, Lck, Hck, and Yes, bind to the C terminus of K15 between amino acids 355 and 373 and phosphorylate the tyrosine residue at position 481 in the $\mathrm{SH} 2$ binding motif (3). 
Recent studies demonstrated that the largest (45-kDa) K15 gene product, which is a protein with 12 membrane-spanning domains, activates the Ras/mitogen-activated protein kinase and NF-кB signal transduction pathways (3). A tumor necrosis factor receptor-associated factor binding site is also present in the carboxy terminus of K15. Tumor necrosis factor receptorassociated factors 1, 2, and 3 bind to the YEEVL motif in the cytoplasmic tail of K15, an interaction required for the activation of $\mathrm{NF}-\kappa \mathrm{B}$ (13). A point mutation at tyrosine residue position $481\left(\mathrm{Y}^{481}\right)$ within the $\mathrm{Y}^{481}$ EEVL motif abolished K15mediated activation of the NF- $\mathrm{KB}$ and Ras/mitogen-activated protein kinase pathways, suggesting that phosphorylation of YEEVL is necessary for signaling (3). In addition, K15 interacts with antiapoptotic protein HAX-1 in vitro and in vivo (27). The YASIL motif within the C terminus of K15 is necessary for interaction with HAX-1. HAX-1 has domains homologous to the apoptosis inhibitor Bcl-2. HAX-1 forms a homodimer in vivo and blocks Bax-induced apoptosis.

$\mathrm{K} 15$ is likely to play a significant role in KSHV-induced pathogenesis. Hence, understanding the mechanism by which K15 expression is regulated may shed light on its role in the KSHV life cycle. In the present study, we have identified the K15-P transcription initiation site by $5^{\prime}$ rapid amplification of cDNA ends (RACE) and RNase protection assay (RPA) and have mapped the K15-P promoter element in a number of different cell lines. We also show that the KSHV immediate-early protein Orf50/Rta transactivates the K15 promoter element.

\section{MATERIALS AND METHODS}

Cell culture. Suspension BCBL-1 cells and BJAB cells were cultured in RPMI 1640 medium (Cellgro) containing L-glutamine, 10\% fetal bovine serum, penicillin $(100 \mathrm{U} / \mathrm{ml})$, and streptomycin $(50 \mu \mathrm{g} / \mathrm{ml})$. For BCBL-1 cells, the medium was further supplemented with $55 \mu \mathrm{M} 2$-mercaptoethanol and $0.0075 \%$ sodium bicarbonate. Adherent 293 epithelial cells were maintained in Dulbecco modified Eagle medium (Sigma) with 10\% fetal bovine serum, 2 mM Glutamax I (Invitrogen), penicillin $(100 \mathrm{U} / \mathrm{ml})$, and streptomycin $(50 \mu \mathrm{g} / \mathrm{ml})$. All cells were grown at $37^{\circ} \mathrm{C}$ in $5 \%$ carbon dioxide $\left(\mathrm{CO}_{2}\right)$

5' RACE. KSHV-positive BCBL-1 cells were pretreated with either the phorbol ester 12-O-tetradecanoylphorbol-13-acetate (TPA) at a final concentration of $25 \mathrm{ng} / \mathrm{ml}$ or an equal volume of dimethyl sulfoxide (DMSO) for 40 to $48 \mathrm{~h}$. TPA was prepared in DMSO at $25 \mu \mathrm{g} / \mathrm{ml}$. Total RNA was isolated from pretreated cells with the RNA STAT-60 kit (Tel-Test, Inc.). Total RNA was further enriched for polyadenylated RNA by using the Oligotex mRNA kit (QIAGEN). Six hundred nanograms of polyadenylated RNA was used for 5' RACE. First-strand cDNA synthesis and 5' RACE were performed as specified in the BD SMART RACE kit protocol (Clontech). Reverse transcription was carried out in the presence and absence of Powerscript reverse transcriptase (Clontech). The K15P-specific primer 5'-AGTAGGGCCCAAAGCCATAAATTCCAGA-3' was used in the appropriate $5^{\prime}$ RACE PCRs. Thermal cycling was run with the following program: (i) $94^{\circ} \mathrm{C}$ for $5 \mathrm{~min}$, (ii) $94^{\circ} \mathrm{C}$ for $5 \mathrm{~s}$, (iii) $65^{\circ} \mathrm{C}$ for $10 \mathrm{~s}$, (iv) $72^{\circ} \mathrm{C}$ for $3 \mathrm{~min}$, (v) repetition of steps ii to iv for $39 \mathrm{cycles}$, and (vi) $70^{\circ} \mathrm{C}$ for 10 min. Amplified cDNA products separated in a $2 \%$ low-melting-point agarose gel were isolated from the gel and cloned into the pCR2.1 TOPO TA vector (Invitrogen TOPO cloning kit). The TOPO clones were transformed into TOP 10 competent cells (Invitrogen) and plated on Luria-Bertani plates supplemented with ampicillin at $100 \mu \mathrm{g} / \mathrm{ml}$. Bacterial colonies were picked, and the isolated DNAs were sequenced with the M13 reverse primer by submission to the University of North Carolina at Chapel Hill Genome Analyses Facility.

RPA. Polyadenylated RNA isolated from BCBL-1 cells pretreated with TPA $(25 \mathrm{ng} / \mathrm{ml})$ or an equal volume of DMSO was used for RPA. RPA was carried out with the Ambion RPA III kit. To generate the K15 probe, a 500-bp fragment was amplified spanning $150 \mathrm{bp}$ of the K15 first ORF and $350 \mathrm{bp}$ upstream from the ATG translation start site. Genomic DNA from KSHV-positive BCBL-1 cells served as the DNA template. The forward and reverse primers, engineered to contain BamHI sites, used for the amplification reactions were 5'-CGGCGG GGATCCAGACTCCTCGCACAGTAGAG-3' and 5'-CGGCGGGGATCCTTA
ATAGCACTGACAAACAA-3', respectively. The amplified fragment was then cloned into the pSP72 vector at the BamHI site. To make an antisense RNA probe, the plasmid was linearized with HindIII. The Promega Riboprobe kit and $\left[\alpha-{ }^{32} \mathrm{P}\right] \mathrm{rCTP}$ were used to transcribe and generate an antisense RNA probe in vitro. The radiolabeled probe was purified with the RNeasy Mini kit (QIAGEN). At least $6 \times 10^{6} \mathrm{cpm}$ of the K15 probe was used for RPA. For the K15 RPA, hybridization of the radiolabeled probe to mRNA was carried out overnight at $55^{\circ} \mathrm{C}$. For the positive control, $\beta$-actin RPA, the manufacturer's protocol was followed. Products were separated on a denaturing $6 \%$ polyacrylamide-urea gel. The ФX174 DNA HinfI-digested marker (Promega) was end labeled with $\left[\gamma^{-32} \mathrm{P}\right] \mathrm{ATP}$ and T4 polynucleotide kinase. The sequencing ladder was prepared from the pSP72 vector and the ${ }^{32} \mathrm{P}$-end-labeled $\mathrm{T} 7$ primer in accordance with the manufacturer's manual (Epicenter Sequitherm Cycle Sequencing kit).

K15 promoter cloning. KSHV-positive BCBL-1 genomic DNA was used as the KSHV DNA template in the PCRs to amplify the sequence upstream of the K15 gene. Primers were designed to contain a HindIII restriction site at the $5^{\prime}$ end and a BglII restriction site at the $3^{\prime}$ end of the amplified product. The following reverse primer was used in all of the PCRs: 5'-CGGGCGGAAGCTTCCTAA GTATTCCCGACACAAG-3'. For upstream promoter truncated mutations, the following forward primers were used: (i) -75K15p (5'-CGGCGGAGATCTTGT TTTTTGCAGCTGTAACAA-3'), (ii) -100K15p (5'-CGGCGGAGATCTTTT CCAGGATACTCTTCATCT-3'), (iii)-125K15p (5'-CGGCGGAGATCTTTT CCAGGATACTCTTCATCT-3'), (iv)-150K15p (5'-CGGCGGAGATCTGGT CCCCCGCGGGCGGGGGCG-3'), (v) -225K15p (5'-CGGCGGAGATCT CCAAACAGGCTCACACACAGC-3'), and (vi) -271K15p (5'-CGGCGG AGATCTCACGTAGTGTCCAGGGCTCCA- $\left.3^{\prime}\right)$. The thermal cycling conditions used were $94^{\circ} \mathrm{C}$ for $10 \mathrm{~min} ; 30$ cycles of $94^{\circ} \mathrm{C}$ for $1 \mathrm{~min}, 55^{\circ} \mathrm{C}$ for $2 \mathrm{~min}$, and $68^{\circ} \mathrm{C}$ for $2 \mathrm{~min}$; and a final $70^{\circ} \mathrm{C}$ for $10 \mathrm{~min}$. PCR products were gel isolated and cloned into the pGL2Basic vector (Promega) at the HindIII and BglII sites. All plasmids were purified from bacterial cultures with the Genelute HP Plasmid Maxiprep kit (Sigma).

Transfections. A 7.5- to $10-\mu \mathrm{g}$ sample of the reporter plasmid, 10 to $15 \mu \mathrm{g}$ of the pCDNA3 or pCDNA3-Orf50/Rta plasmid, and 1 to $4 \mu \mathrm{g}$ of a $\beta$-galactosidase expression plasmid were used for transfection assays. Equal amounts of DNA were transfected into 293 cells by the Lipofectamine Reagent (Invitrogen) method in accordance with the manufacturer's protocol. DNA were electroporated (Bio-Rad Gene Pulser II) at $250 \mathrm{~V}$ and $950 \mu \mathrm{F}$ into $10^{7}$ BJAB or BCBL-1 cells in $400 \mu \mathrm{l}$ of plain RPMI medium in cuvettes with a 4-mm gap (Bio-Rad). After electroporation, cells were allowed to recover for $15 \mathrm{~min}$ in room temperature, followed by transfer into 12-well plates containing $4 \mathrm{ml}$ of complete medium per well. When indicated, TPA $(25 \mathrm{ng} / \mathrm{ml})$ or DMSO (equal volume) was added to the complete medium 4 to $6 \mathrm{~h}$ after electroporation. Cells were incubated at $37^{\circ} \mathrm{C}$ in $5 \% \mathrm{CO}_{2}$ for an additional 40 to $48 \mathrm{~h}$.

Cells were harvested for reporter assays $48 \mathrm{~h}$ after transfection. 293 cells were rinsed three times with phosphate-buffered saline, followed by freeze-thawing once in $200 \mu \mathrm{l}$ of Reporter Lysis Buffer (Promega). BCBL-1 and BJAB cells were rinsed twice in $5 \mathrm{ml}$ of phosphate-buffered saline, followed by freeze-thawing once in $100 \mu \mathrm{l}$ of Reporter Lysis Buffer (Promega). Clarified supernatants were assayed for luciferase activity with the Promega luciferase reagent. $\beta$-Galactosidase activity was determined by using the Galacto-Star kit (Applied Biosystems).

Normalized luciferase activity from each sample was calculated as the number of relative light units (RLU) divided by the number of units of $\beta$-galactosidase activity. Fold activation was reported as normalized luciferase activity from each sample divided by the normalized luciferase activity of cells transfected with the reporter vector alone (pGL2Basic). Since TPA treatment or cotransfection with an ORF50/Rta expression plasmid increased the normalized luciferase activity over that obtained with the pGL2Basic vector alone, the number of RLU plotted on the $y$ axis was calculated as the normalized luciferase activity of each sample minus the background activity of the reporter construct alone. All transfections were carried out in duplicate or triplicate and repeated at least three times. Error bars represent the standard deviation.

\section{RESULTS}

Identification of the K15 transcription initiation site. In order to identify the promoter element of the full-length K15-P gene, mapping its transcription start site was essential. To date, no literature on the transcription initiation site of K15 has been published. Normal BCBL-1 cells contain $\sim 50$ copies of the KSHV genome (40) in the latent state, and when treated with TPA, the viral genome is induced to enter the lytic cycle (19). 


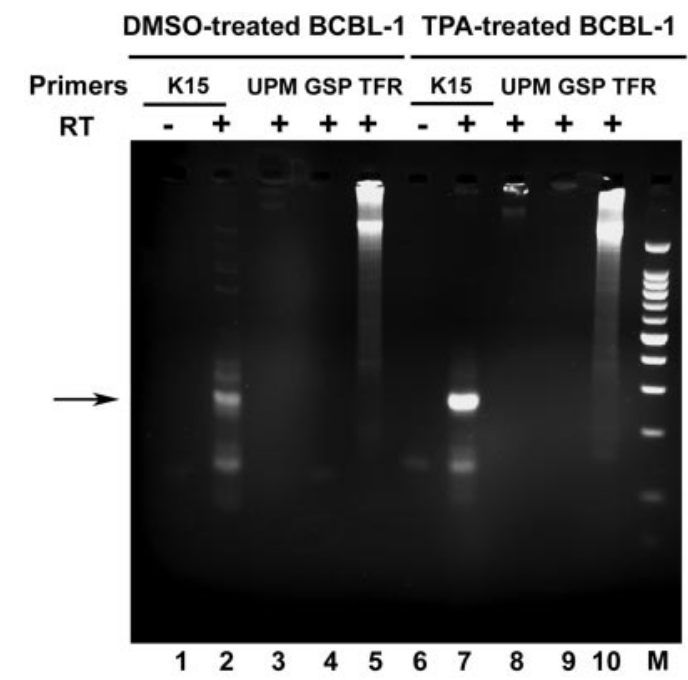

FIG. 1. Mapping of the transcription initiation site of the K15 gene by $5^{\prime}$ RACE. To generate a cDNA copy of the K15-P mRNA transcripts from DMSO- and TPA-treated BCBL-1 cells, reverse transcription was performed with $(+)$ and without $(-)$ reverse transcriptase (RT) and with an oligo(dT) primer. DNA fragments spanning the $5^{\prime}$ end of the K15 transcript were then PCR amplified with a K15-Pspecific reverse primer (GSP) within exon 1 and the forward primer UPM, which anneals to the SMART II A Oligo that was incorporated into the $5^{\prime}$ end of the cDNA during reverse transcription. PCR products were separated on a $1.5 \%$ agarose gel. Shown is an agarose gel depicting PCR products amplified from the mRNA of latent (lane 2) and lytic (lane 7) BCBL-1 cells. Reactions containing a UPM- or K15-specific primer (GSP) only served as negative controls. Positive controls (lanes 5 and 10 ) were reactions which amplified the $5^{\prime}$ end of the transferrin receptor (TFR) transcript from human placental mRNA. M represents a 100-bp ladder (Promega) used as a DNA marker.

K15 is weakly expressed in latently infected B cells but induced in TPA-treated BC3 (15) and BCBL-1 (22) cells. To enrich for K15 transcripts, we sought to map the K15 transcription initiation site with BCBL-1 cells treated with either the vehicle alone (DMSO) or TPA. Briefly, total RNAs from DMSOtreated (latent) and TPA-induced (lytic) BCBL-1 cells were isolated with the Tel-Test RNA Stat-60 kit. RNA was further enriched for polyadenylated $\left[\operatorname{poly}(\mathrm{A})^{+}\right]$transcripts with the QIAGEN Oligotex mRNA kit. 5' RACE was then performed with the 5' RACE kit from Clontech. First-strand cDNAs were generated by reverse transcription from transcripts. The 5' RACE reaction adds an oligonucleotide (SMART II A Oligo) to the $5^{\prime}$ end of the first-strand cDNA. With the forward primer or Universal Primer Mix (UPM) specific to the SMART II A Oligo and a reverse primer specific to K15-P (within exon 1), the 5' RACE reaction yielded a dominant 230-bp PCR product from both latent and lytic cells (Fig. 1, lanes 2 and 7, respectively). The 5' RACE PCR product was further increased in TPA-treated BCBL-1 lytic cells (lane 7 compared to lane 2), as expected. This product was not amplified from genomic DNA, as it was not present in reaction mixtures without reverse transcriptase (lanes 1 and 6). Additional negative controls included PCRs with the UPM and GSP primers only (Fig. 1, lanes 3 and 4 and lanes 8 and 9). Positive controls for the 5' RACE assay were represented in lanes 5 and 10 as the amplified $5^{\prime}$ end of the transferrin re- ceptor transcript from human placental mRNA. The 230-bp PCR product from latent and lytic cells was gel isolated and cloned into the pCR2.1-TOPO vector (Invitrogen). Several clones were sequenced for each construct, and the DNA sequences of these clones revealed a K15-P transcription initiation site approximately 152 bp upstream from the ATG translation start site in both latently and lytically infected BCBL-1 cells.

The nucleotide position at which transcription commences was confirmed by RPA. Briefly, a 500-bp 5' K15 probe extending from $-350 \mathrm{bp}$ to $+150 \mathrm{bp}$ relative to the ATG translation start site, encompassing the first $150 \mathrm{bp}$ of the K15 ORF, was synthesized by PCR from KSHV genomic DNA isolated from BCBL-1 cells. BamHI sites were engineered into the primers, and the PCR product was cloned into the $\mathrm{pSP} 72$ vector, which contains the T7 RNA polymerase transcription initiation site. An antisense 5' K15 probe was transcribed in vitro from the linearized plasmid and radiolabeled with $\left[\alpha-{ }^{32} \mathrm{P}\right] \mathrm{CTP}$. Overnight hybridization of the antisense probe to poly $(\mathrm{A})^{+} \mathrm{RNA}$ isolated from TPA- or DMSO-treated BCBL-1 cells was performed, followed by RNase $\mathrm{A}-\mathrm{T}_{1}$ digestion of unprotected RNA fragments. It is important to note that $50 \mu \mathrm{g}$ of mRNA harvested from BCBL-1 cells pretreated with TPA (T, lane 8) or $100 \mu \mathrm{g}$ of DMSO (D, lane 9), i.e., twice as much mRNA, was used in the RNase digestion. The protected probe fragments were separated on a $6 \%$ denaturing polyacrylamideurea gel, and the size of the protected fragment remaining after RNase digestion was determined by comparison to a known DNA sequence ladder. Figure 2 shows the result of the RPA. The K15 RPA yielded a 302-nucleotide protected fragment, as indicated by the symbol 0 . The transcript is present in both TPA-treated BCBL-1 cells (lane 8) and DMSO-treated latently infected cells (lane 9). A sequencing ladder (lanes 11 to 14) was also run on the same gel (Fig. 2). As the exact position of the chosen antisense probe was known, the K15 transcription initiation site was confirmed at position 152 upstream from the ATG translation start site of K15. This is the same site obtained in the 5' RACE experiment described above. Positive controls for the RPA included a $\beta$-actin probe which yielded a protected fragment from liver mRNA (Fig. 2, lanes 2 to 4$)$.

K15 promoter basal activity. Elucidation of the cis-acting elements within the K15 promoter will provide important insights into the regulatory mechanisms of K15 gene expression. Figure 3 displays the upstream sequence from the K15 ATG translation start site to the end of the sequenced BCBL genome (GenBank accession no. U85269) (40). The GC-rich terminal repeats start at 270 nucleotides upstream of the ATG translation site, as indicated by an asterisk (GenBank accession no. U85269) (40). The translation start site is marked by a black arrow and the transcription start site by a white arrow (Fig. 3). The sequence was scanned with the Genomatix MatInspector program to identify putative transcription factor binding sites. There appears to be a very weak TATA box (TATTTAT) about 25 nucleotides upstream of the K15 transcription initiation site. Some of the transcription factor binding sites in the K15 promoter include Oct1, E2F, Sp1, NFкB, Stat1, and Smad3.

To examine the cis-acting elements necessary to activate the K15 gene, a series of 5' -truncated promoter deletion constructs 


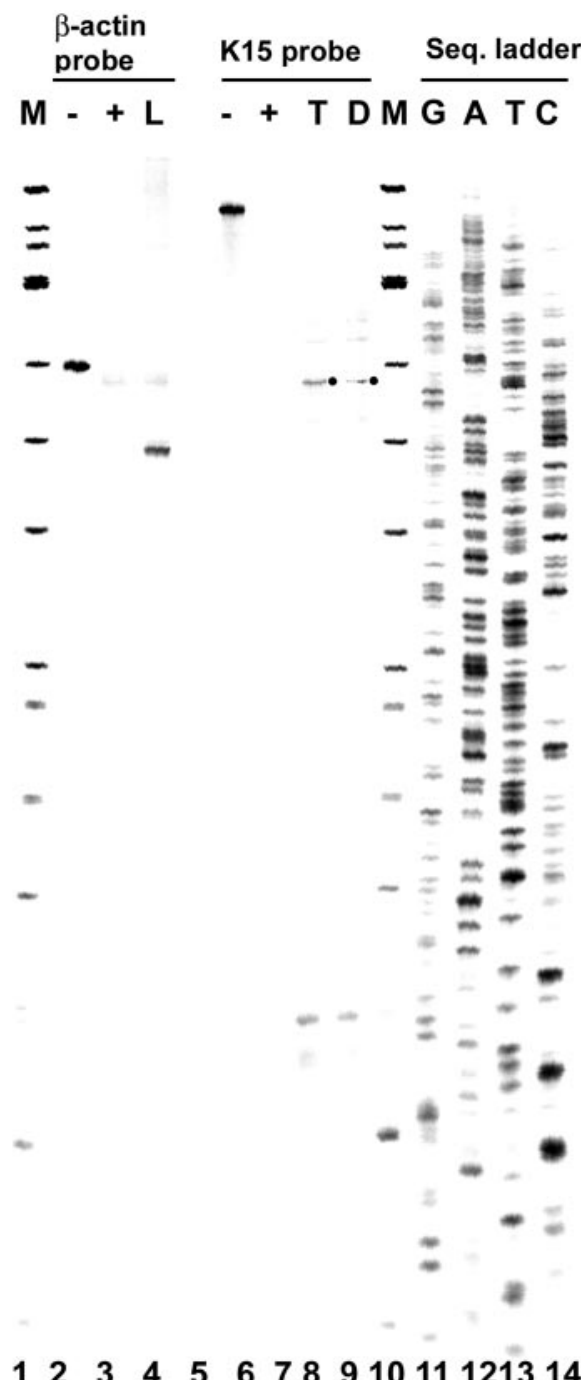

FIG. 2. RPA of the K15-P transcript. A 50- or 100- $\mu$ g sample of mRNA harvested from BCBL-1 cells pretreated with TPA (T, lane 8) or DMSO (D, lane 9), respectively, for $48 \mathrm{~h}$ was hybridized overnight with an antisense radiolabeled K15 riboprobe, followed by RNase A- $T_{1}$ digestion. The protected probe fragment was separated on a $6 \%$ denaturing polyacrylamide-urea gel. Lanes 8 and 9 show the 302nucleotide protected fragment $(-)$ representing the K15 transcript in TPA- (T) or DMSO (D)-treated BCBL-1 cells, respectively. Lane 6 $(-)$ represents the undigested K15 probe. Lane $7(+)$ represents the probe digested with RNase. The marker (M, lanes 1 and 10) is a radiolabeled $\Phi$ X174/HinfI DNA marker (Promega). Lanes 2 to 4 contain positive controls consisting of the $\beta$-actin probe hybridized with total liver RNA (supplied with the Ambion RPA III kit). Lane $2(-)$ represents the undigested $\beta$-actin probe, and lane $3(+)$ represents the $\beta$-actin probe digested with RNase. Lanes 11 through $14(\mathrm{G}, \mathrm{A}, \mathrm{T}$, and $\mathrm{C}$, respectively) represent a sequencing (Seq.) ladder.

were synthesized. Fragments containing sequences from the K15 transcription initiation site to $75,100,125,150,225$, and 271 bp upstream were generated by PCR with genomic DNA from KSHV-positive BCBL-1 cells as the DNA template. These PCR-generated fragments were subcloned in front of the luciferase reporter gene in the pGL2Basic vector. Figure 4 illustrates the six deletion constructs. The nomenclature for each plasmid is listed on the right. The $-125 \mathrm{~K} 15 \mathrm{p},-150 \mathrm{~K} 15 \mathrm{p}$,

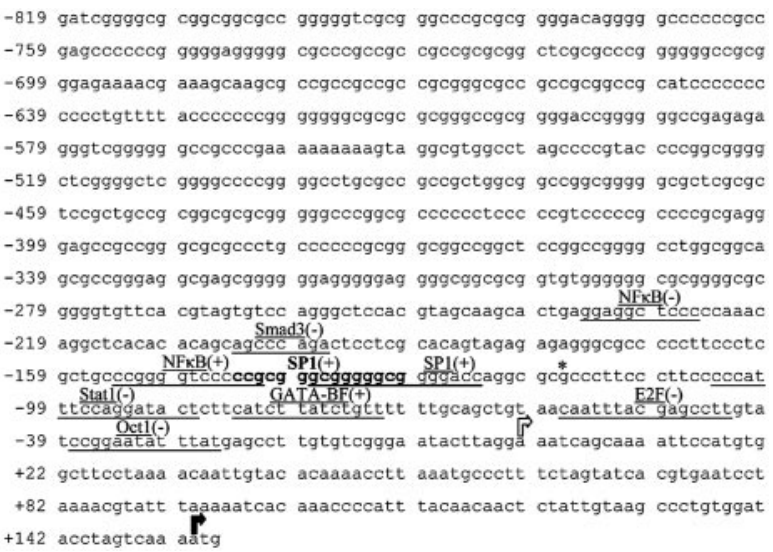

FIG. 3. Identification of the K15 promoter. Sequences upstream of the K15 transcription initiation site (white arrow) of the BCBL-1 KSHV genome were scanned with the MatInspector program from Genomatix. Transcription binding sites are in bold or underlined. The symbols + and - represent the sense strand and antisense strand, respectively. The asterisk marks the start of the $5^{\prime}$ high-GC-content terminal repeats. The black arrow represents the ATG translation initiation codon, and the white arrow represents the transcriptional start site.

$-225 \mathrm{~K} 15 \mathrm{p}$, and $-271 \mathrm{~K} 15 \mathrm{p}$ promoters contain sequences within the terminal repeats.

To investigate the ability of each of the K15 deletion promoter elements to drive luciferase expression, reporter assays were carried out with various cell lines. The K15 promoterreporter plasmids were each transiently transfected into 293 human kidney epithelial cells with Lipofectamine (Invitrogen) and into BJAB and BCBL-1 cells by electroporation. Cotransfection with constant amounts of a $\beta$-galactosidase reporter plasmid was used to normalize for transfection efficiency. The transfections were performed in duplicate and repeated numerous times. Figure 5 shows the results of these reporter assays. Transfection assays with KSHV-negative 293 cells (Fig. 5A) and BJAB cells (Fig. 5B) demonstrate 15- to 35-fold activation of the K15 promoter constructs over that of the empty vector (pGL2basic) in both cell lines. Only the $-75 \mathrm{~K} 15 \mathrm{p}$ promoter containing the first 75-bp sequence from the transcription ini-

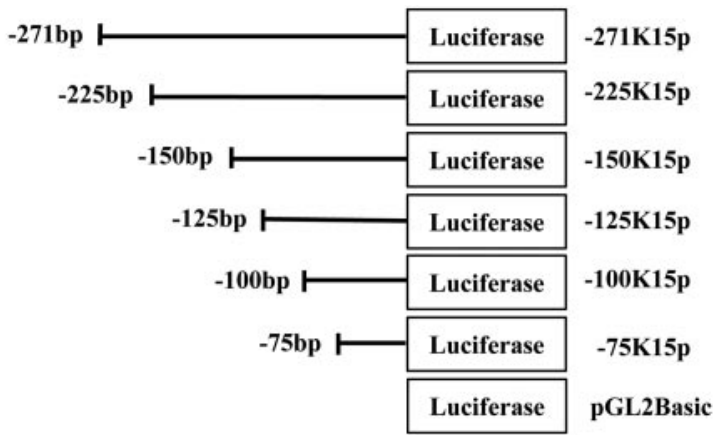

FIG. 4. K15 promoter deletion constructs. The $5^{\prime}$ end of the K15 promoter was serially truncated to contain $271 \mathrm{bp}, 225 \mathrm{bp}, 150 \mathrm{bp}, 125$ bp, $100 \mathrm{bp}$, or $75 \mathrm{bp}$ upstream of the K15 transcription initiation site. Each fragment was subcloned into the pGL2Basic vector upstream of the luciferase reporter gene. The name assigned to each construct is shown on the right. 
A

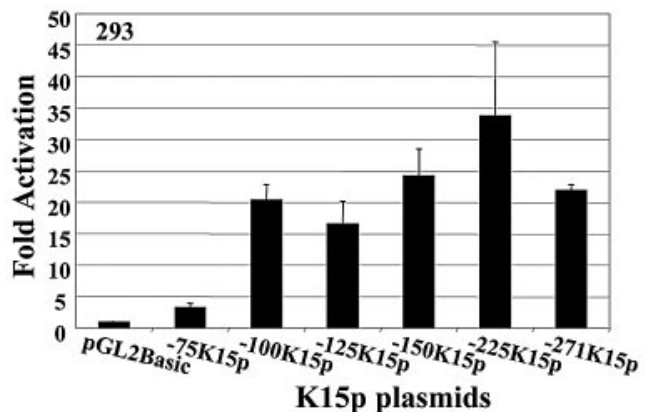

B
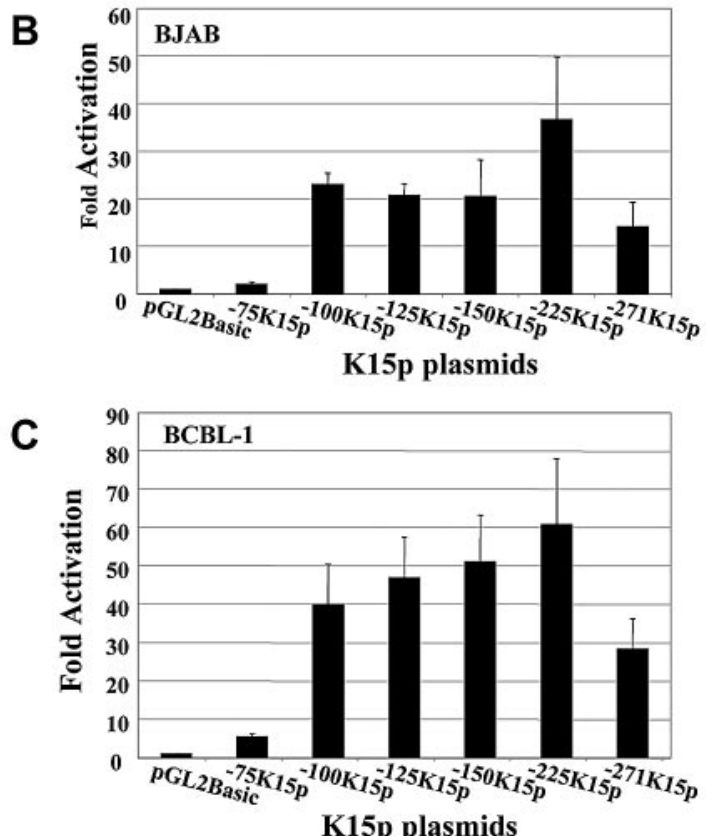

FIG. 5. Basal activity of the K15 promoter. A 7.5- to 10- $\mu$ g sample of each of the K15 promoter constructs and 2 to $4 \mu \mathrm{g}$ of a $\beta$-galactosidase expression plasmid were transfected into KSHV-negative $293 \mathrm{kid}-$ ney epithelial cells (A), KSHV-negative BJAB cells (B), and KSHVpositive BCBL-1 cells (C). Cells were harvested $48 \mathrm{~h}$ posttransfection and assayed for luciferase activity (RLU). Relative activation ( $n$-fold) was calculated as normalized luciferase activity (RLU/ $\beta$-galactosidase activity) divided by the activity of cells transfected with the pGL2Basic reporter construct alone. Error bars represent the standard deviation of duplicate samples.

tiation site showed minimal activity in both 293 and BJAB cells. A similar profile was seen in KSHV-positive BCBL-1 cells (Fig. 5C). The $-100 \mathrm{~K} 15 \mathrm{p},-125 \mathrm{~K} 15 \mathrm{p},-150 \mathrm{~K} 15 \mathrm{p},-225 \mathrm{~K} 15 \mathrm{p}$, and $-271 \mathrm{~K} 15 \mathrm{p}$ promoter elements displayed basal activity 30 to 60 -fold higher than that of the empty vector. These results suggest that the minimum sequence that can function as a promoter element for K15 gene expression contains the first 100 nucleotides upstream of the K15 transcription start site.

TPA-induced lytic infection activates the K15 promoter elements. The K15 gene is weakly expressed in latent BCBL-1 cells, but expression increases after chemical induction of viral lytic replication $(8,13,23)$, thus implying differential transcriptional regulation at variable stages of the viral cycle and/or the host environment. We investigated whether lytic induction with TPA could activate K15 gene expression. The two mini-
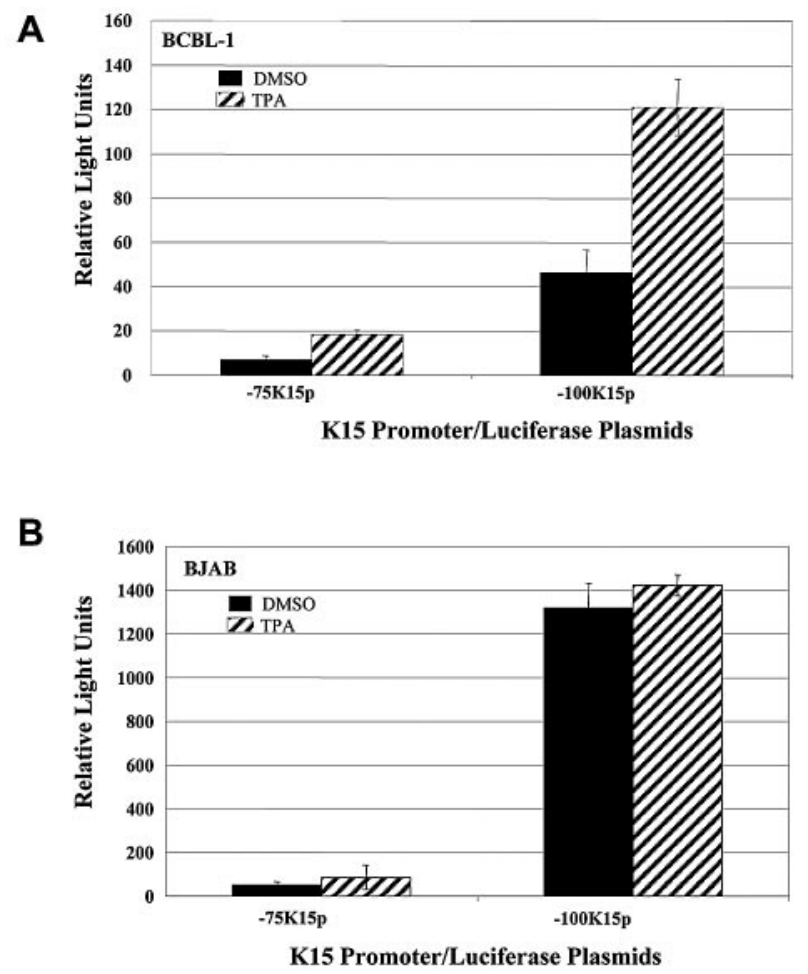

FIG. 6. Analysis of K15 promoter activity in TPA-treated B cells. A $10-\mu \mathrm{g}$ sample of each of the $-75 \mathrm{~K} 15 \mathrm{p}$ and $-100 \mathrm{~K} 15 \mathrm{p}$ promoter constructs and 2 to $4 \mu \mathrm{g}$ of a $\beta$-galactosidase expression plasmid were transfected into KSHV-positive BCBL-1 cells (A) and KSHV-negative $\mathrm{BJAB}$ cells (B). At 4 to $6 \mathrm{~h}$ posttransfection, cells were treated with TPA at $25 \mathrm{ng} / \mathrm{ml}$ (hatched bars) or an equal volume of DMSO (black bars). Cells were harvested $48 \mathrm{~h}$ posttransfection and assayed for luciferase activity (RLU). Promoter activity (RLU) was calculated as normalized luciferase activity (RLU/ $\beta$-galactosidase activity) minus the background activity of cells transfected with the pGL2Basic reporter construct alone. Error bars represent the standard deviation of duplicate samples.

mal promoter element $-75 \mathrm{~K} 15 \mathrm{p}$ and $-100 \mathrm{~K} 15 \mathrm{p}$ constructs were transfected into BJAB and BCBL-1 cells by electroporation. Four to $6 \mathrm{~h}$ after electroporation, cells were treated with either TPA $(25 \mathrm{ng} / \mathrm{ml})$ or an equal volume of DMSO. Cells were harvested $45 \mathrm{~h}$ posttransfection, and luciferase assays were performed. Figure 6A shows that addition of TPA to KSHV-positive BCBL-1 cells induced both the $-75 \mathrm{~K} 15 \mathrm{p}$ and $-100 \mathrm{~K} 15 \mathrm{p}$ promoter elements 2.5 -fold over those in DMSOtreated cells. In contrast, neither the $-75 \mathrm{~K} 15 \mathrm{p}$ nor the $-100 \mathrm{~K} 15 \mathrm{p}$ promoter element was activated in TPA-treated, KSHV-negative BJAB cells (Fig. 6B). The difference between these two cell lines implies that TPA-mediated transcriptional activation of the two minimal K15 promoter elements is dependent on a KSHV protein present in BCBL-1 cells.

KSHV Orf50/Rta activates K15 promoter elements. Since our study showed that activation of the two K15 minimal promoter elements, $-75 \mathrm{~K} 15 \mathrm{p}$ and $-100 \mathrm{~K} 15 \mathrm{p}$, by TPA induction to lytic replication was KSHV dependent, we sought to identify viral lytic gene products involved in K15 transactivation. KSHV immediate-early protein Orf50/Rta was a primary candidate. Orf50/Rta is a potent transactivator of early and late viral genes, thus triggering the cascade of viral replication. The 
A -100

TTTCCAGGATACTCTTCATCTTAT

-75 CTGTTTTTTGCAGCTGTAACAATTTACGAGCCTTGTAT

-39 CCGGAATATTATGAGCCTTGTGTCGgGATACTTAGGA
C

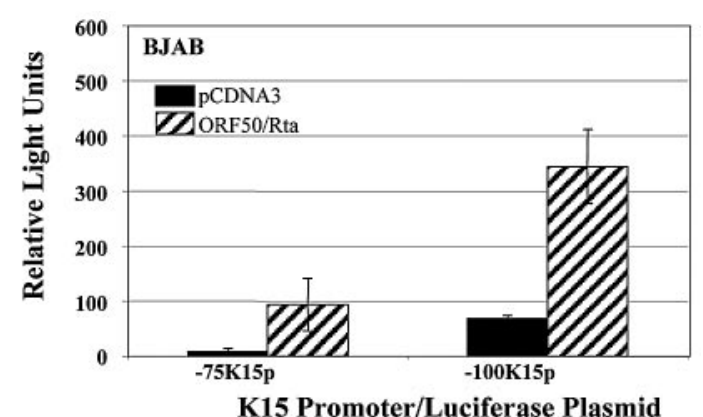

D

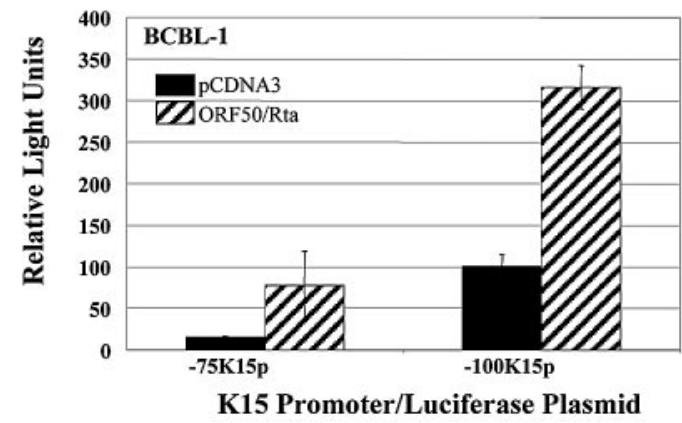

FIG. 7. Orf50/Rta activates the K15 promoter. (A) Analysis of the $-100 \mathrm{~K} 15$ promoter element. The K15 promoter construct, $-100 \mathrm{~K} 15 \mathrm{p}$ containing 100 nucleotides relative to the transcription start site (white arrow), was scanned for RREs. Underlined letters represent the consensus RRE motif, A/T triplets separated by 7 or 17 nucleotides, respectively (16). (B) A 7.5- $\mu \mathrm{g}$ sample of each of the $-75 \mathrm{~K} 15$ and $-100 \mathrm{~K} 15 \mathrm{p}$ promoter constructs and $1 \mu \mathrm{g}$ of a $\beta$-galactosidase expression plasmid were cotransfected with $15 \mu \mathrm{g}$ of the pCDNA3 vector (black bars) or the pCDNA3-Orf50/Rta plasmid (hatched bars) into KSHV-negative 293 cells. (C) A 10- $\mu$ g sample of each of the $-75 \mathrm{~K} 15$ and - 100K15p promoter constructs and 2 to $4 \mu \mathrm{g}$ of a $\beta$-galactosidase expression plasmid were cotransfected with $10 \mu \mathrm{g}$ of the pCDNA3 vector (black bars) or the pCDNA3-Orf50/Rta plasmid (hatched bars) into KSHV-negative BJAB cells. (D) The same method as described for panel C was used to transfect KSHV-positive BCBL-1 cells. In panels B through D, cells were harvested $48 \mathrm{~h}$ posttransfection and assayed for luciferase activity (RLU). Promoter activity (RLU) was calculated as normalized luciferase activity (RLU/ $\beta$-galactosidase activity) minus the background activity of cells transfected with the pGL2Basic reporter construct alone. Error bars represent the standard deviation of duplicate samples.

KSHV Orf50/Rta protein has been previously shown to activate a number of different downstream KSHV promoters, including nut-1, ORF57, K8, K1, and vIL-6 (2, 11, 17, 29-31, 36). Orf50/Rta recognizes and binds diverse DNA sequence motifs in responsive promoters. A number of Rta-responsive elements (RREs) have been identified $(6,11,12,16,25,29)$. The consensus RRE includes a pattern of multiple $\mathrm{A} / \mathrm{T}$ triplets repeated every 7 or 17 nucleotides with high-GC sequences preferred for the spacer (16) (Fig. 7A). To determine the effects of Orf50/Rta on the K15 promoter, the empty vector pGL2Basic and the K15 promoter deletion construct -75K15p and $-100 \mathrm{~K} 15 \mathrm{p}$ promoter-luciferase plasmids were each transfected into 293, BJAB, and, BCBL-1 cells with or without the Orf50/Rta expression plasmid (Fig. 7B, C, and D). In 293 cells, Orf50/Rta activated $-75 \mathrm{~K} 15 \mathrm{p}$ 13-fold and -100K15p 3-fold over the activity of cells cotransfected with pCDNA3 (empty vector) (Fig. 7B). Similarly, in BJAB cells, $-75 \mathrm{~K} 15 \mathrm{p}$ and $-100 \mathrm{~K} 15 \mathrm{p}$ were activated by Orf50/Rta 4.5 -fold and 5 -fold, respectively (Fig. 7C). In BCBL-1 cells, Orf50/Rta increased the activation of $-75 \mathrm{~K} 15 \mathrm{p}$ and $-100 \mathrm{~K} 15 \mathrm{p}$ fivefold and threefold, respectively, over cotransfection with pCDNA3 (Fig. 7D). Thus, the immediate-early gene product Orf50/Rta can activate transcription of the K15 promoter element.

\section{DISCUSSION}

In this study, we have identified, for the first time, the major transcription start site of the full-length K15 KSHV gene. We utilized both 5' RACE and RPAs to confirm the transcription start site of the K15 gene. Both 5' RACE and RPA mapped the K15 transcription initiation site to 152 nucleotides upstream from the K15 translation start site (ATG). Most eukaryotic promoters contain a TATA box element located 25 to $30 \mathrm{bp}$ upstream from the transcription start site. We have identified a putative weak TATA box, TATTTAT, located 26 bp upstream of the K15 transcription initiation site. Cellular transcription binding sites in the K15 promoter include Oct-1, E2F, Stat-1, Sp1, and NFK-B.

Interestingly, we compared the K15 promoter sequences of four different isolates of KSHV which encode the predominant, K15-P, form of the protein: the BCBL-R isolate from PEL cells (accession number U85269), the GK18 isolate (accession number AF148805) from KS tumors, and two Ugandan K15-P isolates (accession numbers AY042968 and AY042973). The promoter element we have mapped for K15-P was 100\% conserved among all of these viral genomes (Fig. 8), suggesting that K15-P transcription is regulated by factors that are conserved among these different KSHV genomes. However, the 


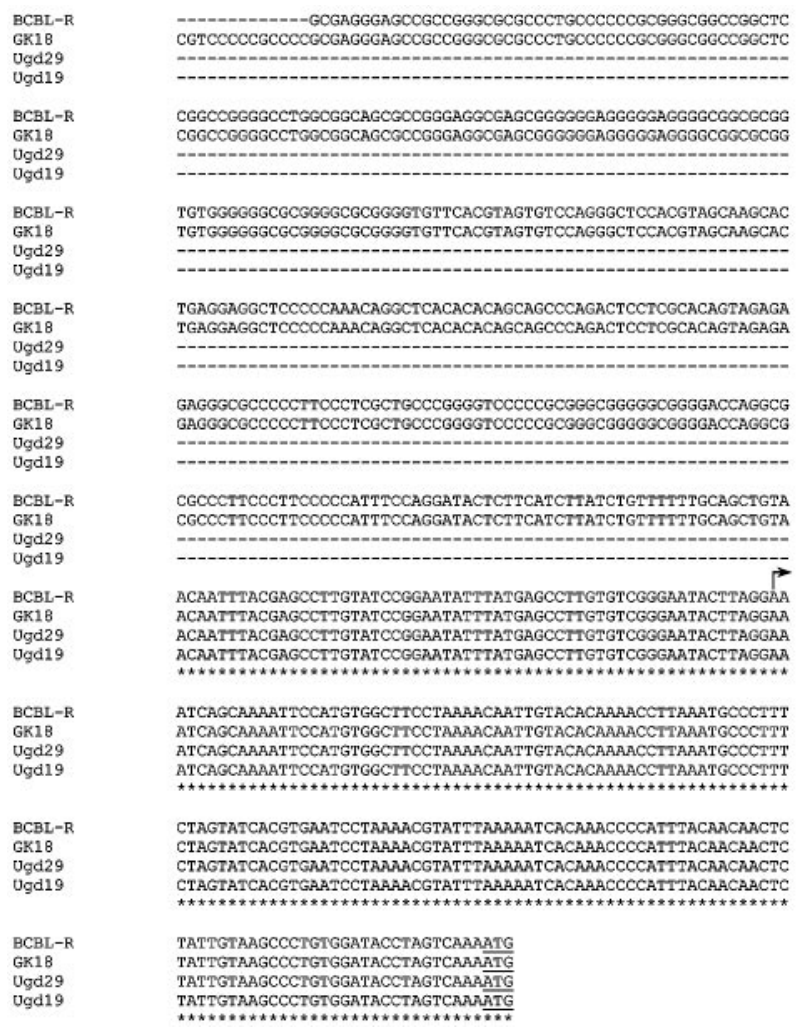

FIG. 8. Alignment of K15-P promoter sequences. K15-P sequences from the BCBL-R isolate from PEL cells (accession number U85269), the GK18 isolate (accession number AF148805) from KS tumors, and two Ugandan K15-P isolates (accession numbers AY042968 and AY042973) were aligned with the ClustalW program. Less of the upstream sequence was available for the two Ugandan K15 isolates. The arrow depicts the K15-P transcriptional start site, while the underlined ATG codon represents the translational start site of K15-P.

$\mathrm{BC}-1$ genome, which encodes the $\mathrm{K} 15-\mathrm{M}$ protein, is more divergent at both the promoter and protein levels. The K15-P and K15-M protein isoforms display less than $30 \%$ identity at the amino acid level (23). Although it is possible that transcriptional regulation of the K15-M protein may be significantly different, we hypothesize that the K15-M promoter may also be regulated by Orf50/Rta in a fashion similar to that of the K15-P promoter.

Transfection reporter assays demonstrated that basal expression of full-length K15-P occurs in both KSHV-negative 293 and BJAB cells and in KSHV-positive BCBL-1 cells. Most of the K15 promoter constructs were activated about 20 -fold in 293 and BJAB cells and about 40-fold in BCBL-1 cells. The minimal sequence sufficient for the basal transcription of K15 lies in the first $100 \mathrm{bp}$ upstream of the transcription initiation site.

The basal transcription of K15 was approximately twofold higher in KSHV-positive cells than in KSHV-negative cells, suggesting that the increased expression of K15 was virus mediated. TPA-induced lytic replication increased K15 transcription, as seen in the 5' RACE, RPA, and reporter assays. Induction of K15 could be secondary to TPA itself and/or due to KSHV. Our data showed that TPA activated the $-75 \mathrm{~K} 15 \mathrm{p}$ and $-100 \mathrm{~K} 15$ p promoter elements in KSHV-positive BCBL-1 cells but not in KSHV-negative BJAB cells, implying that KSHV proteins play a role in activating K15 expression.

The KSHV immediate-early gene product, Orf50/Rta, is known to transactivate several downstream KSHV promoters, including nut-1, ORF57, K8, K1, and vIL-6 (2, 10, 17, 29-31), and our data support adding the K15 promoter to this set. Cotransfection experiments showed that both the $-75 \mathrm{~K} 15 \mathrm{p}$ and $-100 \mathrm{~K} 15 \mathrm{p}$ promoter elements were activated by Orf50/ Rta compared to the vector alone in both KSHV-negative and KSHV-positive cells. Transcriptional activation by Orf50/Rta was not surprising since both the $-75 \mathrm{~K} 15 \mathrm{p}$ and $-100 \mathrm{~K} 15 \mathrm{p}$ promoter elements contain multiple consensus RREs. There are two $(\mathrm{A} / \mathrm{T})_{3} \mathrm{~N}_{7}(\mathrm{~A} / \mathrm{T})_{3}$ and six $(\mathrm{A} / \mathrm{T})_{3} \mathrm{~N}_{17}(\mathrm{~A} / \mathrm{T})_{3} \mathrm{RRE}$ motifs, some of which overlap, spanning the 75-bp region upstream from the $\mathrm{K} 15$ transcription start site. One additional $(\mathrm{A} / \mathrm{T})_{3} \mathrm{~N}_{7}(\mathrm{~A} / \mathrm{T})_{3}$ motif and three $(\mathrm{A} / \mathrm{T})_{3} \mathrm{~N}_{17}(\mathrm{~A} / \mathrm{T})_{3}$ motifs are present in the $-100 \mathrm{~K} 15 \mathrm{p}$ promoter element. No RRE sequences were identified outside the 100-bp region immediately upstream of the K15 initiation site. Thus, both our reporter assays and identifications of multiple RRE motifs within $-75 \mathrm{~K} 15 \mathrm{p}$ and $-100 \mathrm{~K} 15 \mathrm{p}$ strongly support Orf50/Rta-mediated transcriptional activation of the K15 gene.

Elucidation of the transcription initiation site and the cisacting elements within the K15-P promoter imparts important insights into the regulatory mechanisms of K15 gene expression. This study will provide the basis for future investigations.

\section{ACKNOWLEDGMENTS}

We thank Stuart Krall for assistance with the cell lines. We also thank members of the Damania lab for informative discussions and D. Dittmer for manuscript reading.

This work was supported by the American Association for Cancer Research (AACR), American Heart Association grant 0640041N, and NIH grant CA096500 to B.D. E.L.W. is supported by NIH training grant 2T32AI007001-29, and B.D. is a Leukemia \& Lymphoma Society Scholar.

\section{REFERENCES}

1. Ablashi, D. V., L. G. Chatlynne, J. E. Whitman, Jr., and E. Cesarman. 2002. Spectrum of Kaposi's sarcoma-associated herpesvirus, or human herpesvirus 8, diseases. Clin. Microbiol. Rev. 15:439-464.

2. Bowser, B. S., S. M. DeWire, and B. Damania. 2002. Transcriptional regulation of the K1 gene product of Kaposi's sarcoma-associated herpesvirus. J. Virol. 76:12574-12583.

3. Brinkmann, M. M., M. Glenn, L. Rainbow, A. Kieser, C. Henke-Gendo, and T. F. Schulz. 2003. Activation of mitogen-activated protein kinase and NF-кB pathways by a Kaposi's sarcoma-associated herpesvirus K15 membrane protein. J. Virol. 77:9346-9358.

4. Cattani, P., G. Nanni, R. Graffeo, M. Capuano, F. Cerimele, I. L. La Parola, A. Diociaiuti, U. Pozzetto, D. Cerimele, G. Fadda, and M. Castagneto. 2000. Pretransplantation human herpes virus 8 seropositivity as a risk factor for Kaposi's sarcoma in kidney transplant recipients. Transplant. Proc. 32:526527.

5. Cesarman, E., Y. Chang, P. S. Moore, J. W. Said, and D. M. Knowles. 1995. Kaposi's sarcoma-associated herpesvirus-like DNA sequences in AIDS-related body-cavity-based lymphomas. N. Engl. J. Med. 332:1186-1191.

6. Chang, P. J., D. Shedd, L. Gradoville, M. S. Cho, L. W. Chen, J. Chang, and G. Miller. 2002. Open reading frame 50 protein of Kaposi's sarcoma-associated herpesvirus directly activates the viral PAN and K12 genes by binding to related response elements. J. Virol. 76:3168-3178.

7. Chang, Y., E. Cesarman, M. S. Pessin, F. Lee, J. Culpepper, D. M. Knowles, and P. S. Moore. 1994. Identification of herpesvirus-like DNA sequences in AIDS-associated Kaposi's sarcoma. Science 266:1865-1869.

8. Choi, J.-K., B.-S. Lee, S. N. Shim, M. Li, and J. U. Jung. 2000. Identification of the novel K15 gene at the rightmost end of the Kaposi' sarcoma-associated herpesvirus genome. J. Virol. 74:436-446.

9. Damania, B., J.-K. Choi, and J. U. Jung. 2000. Signaling activities of gammaherpesvirus membrane proteins. J. Virol. 74:1593-1601.

10. Deng, H., J. T. Chu, M. B. Rettig, O. Martinez-Maza, and R. Sun. 2002. Rta 
of the human herpesvirus 8/Kaposi sarcoma-associated herpesvirus up-regulates human interleukin-6 gene expression. Blood 100:1919-1921.

11. Deng, H., M. J. Song, J. T. Chu, and R. Sun. 2002. Transcriptional regulation of the interleukin- 6 gene of human herpesvirus 8 (Kaposi's sarcoma-associated herpesvirus). J. Virol. 76:8252-8264.

12. Duan, W., S. Wang, S. Liu, and C. Wood. 2001. Characterization of Kaposi's sarcoma-associated herpesvirus/human herpesvirus-8 ORF57 promoter. Arch. Virol. 146:403-413.

13. Glenn, M., L. Rainbow, F. Aurad, A. Davison, and T. F. Schulz. 1999 Identification of a spliced gene from Kaposi's sarcoma-associated herpesvirus encoding a protein with similarities to latent membrane proteins 1 and 2A of Epstein-Barr virus. J. Virol. 73:6953-6963.

14. Horenstein, M. G., R. G. Nador, A. Chadburn, E. M. Hyjek, G. Inghirami, D. M. Knowles, and E. Cesarman. 1997. Epstein-Barr virus latent gene expression in primary effusion lymphomas containing Kaposi's sarcomaassociated herpesvirus/human herpesvirus-8. Blood 90:1186-1191.

15. Jenner, R. G., M. M. Alba, C. Boshoff, and P. Kellam. 2001. Kaposi's sarcoma-associated herpesvirus latent and lytic gene expression as revealed by DNA arrays. J. Virol. 75:891-902.

16. Liao, W., Y. Tang, Y. L. Kuo, B. Y. Liu, C. J. Xu, and C. Z. Giam. 2003 Kaposi's sarcoma-associated herpesvirus/human herpesvirus 8 transcriptional activator Rta is an oligomeric DNA-binding protein that interacts with tandem arrays of phased A/T-trinucleotide motifs. J. Virol. 77:9399-9411.

17. Lukac, D. M., L. Garibyan, J. R. Kirshner, D. Palmeri, and D. Ganem. 2001 DNA binding by Kaposi's sarcoma-associated herpesvirus lytic switch protein is necessary for transcriptional activation of two viral delayed early promoters. J. Virol. 75:6786-6799.

18. Lukac, D. M., R. Renne, J. R. Kirshner, and D. Ganem. 1998. Reactivation of Kaposi's sarcoma-associated herpesvirus infection from latency by expression of the ORF 50 transactivator, a homolog of the EBV R protein. Virology 252:304-312.

19. Miller, G., L. Heston, E. Grogan, L. Gradoville, M. Rigsby, R. Sun, D. Shedd, V. M. Kushnaryov, S. Grossberg, and Y. Chang. 1997. Selective switch between latency and lytic replication of Kaposi's sarcoma herpesvirus and Epstein-Barr virus in dually infected body cavity lymphoma cells. J. Virol 71:314-324.

20. Moore, P. S., and Y. Chang. 2003. Kaposi's sarcoma-associated herpesvirus immunoevasion and tumorigenesis: two sides of the same coin? Annu. Rev. Microbiol. 57:609-639.

21. Nador, R. G., E. Cesarman, A. Chadburn, D. B. Dawson, M. Q. Ansari, J. Sald, and D. M. Knowles. 1996. Primary effusion lymphoma: a distinct clinicopathologic entity associated with the Kaposi's sarcoma-associated herpes virus. Blood 88:645-656.

22. Paulose-Murphy, M., N. K. Ha, C. Xiang, Y. Chen, L. Gillim, R. Yarchoan, P. Meltzer, M. Bittner, J. Trent, and S. Zeichner. 2001. Transcription program of human herpesvirus 8 (Kaposi's sarcoma-associated herpesvirus). J. Virol. 75:4843-4853.

23. Poole, L. J., J. C. Zong, D. M. Ciufo, D. J. Alcendor, J. S. Cannon, R Ambinder, J. M. Orenstein, M. S. Reitz, and G. S. Hayward. 1999. Comparison of genetic variability at multiple loci across the genomes of the major subtypes of Kaposi's sarcoma-associated herpesvirus reveals evidence for recombination and for two distinct types of open reading frame $\mathrm{K} 15$ alleles at the right-hand end. J. Virol. 73:6646-6660.

24. Russo, J. J., R. A. Bohenzky, M. C. Chien, J. Chen, M. Yan, D. Maddalena, J. P. Parry, D. Peruzzi, I. S. Edelman, Y. Chang, and P. S. Moore. 1996. Nucleotide sequence of the Kaposi sarcoma-associated herpesvirus (HHV8). Proc. Natl. Acad. Sci. USA 93:14862-14867.
25. Sakakibara, S., K. Ueda, J. Chen, T. Okuno, and K. Yamanishi. 2001 Octamer-binding sequence is a key element for the autoregulation of Kaposi's sarcoma-associated herpesvirus ORF50/Lyta gene expression. J. Virol. 75: 6894-6900.

26. Seckin, D., A. Demirag, N. Hizel, B. Demirhan, and R. Erdal. 2000. Absence of Epstein-Barr virus DNA sequences in iatrogenic Kaposi's sarcomas of renal transplant recipients. Transplant. Proc. 32:554-555.

27. Sharp, T. V., H. W. Wang, A. Koumi, D. Hollyman, Y. Endo, H. Ye, M. Q. Du, and C. Boshoff. 2002. K15 protein of Kaposi's sarcoma-associated herpesvirus is latently expressed and binds to HAX-1, a protein with antiapoptotic function. J. Virol. 76:802-816.

28. Sitas, F., L. Taylor, J. Madhoo, K. Cooper, H. Carrara, C. Boshoff, and R. A. Weiss. 1997. Occurrence of human herpes virus 8 in Kaposi's sarcoma and other tumours in South Africa. S. Afr. Med. J. 87:1020-1022.

29. Song, M. J., H. J. Brown, T.-T. Wu, and R. Sun. 2001. Transcription activation of polyadenylated nuclear RNA by Rta in human herpesvirus 8/Kaposi's sarcoma-associated herpesvirus. J. Virol. 75:3129-3140.

30. Song, M. J., H. Deng, and R. Sun. 2003. Comparative study of regulation of RTA-responsive genes in Kaposi's sarcoma-associated herpesvirus/human herpesvirus 8. J. Virol. 77:9451-9462.

31. Song, M. J., X. Li, H. J. Brown, and R. Sun. 2002. Characterization of interactions between RTA and the promoter of polyadenylated nuclear RNA in Kaposi's sarcoma-associated herpesvirus/human herpesvirus 8 . J. Virol. 76:5000-5013.

32. Soulier, J., L. Grollet, E. Oksenhendler, P. Cacoub, D. Cazals-Hatem, P. Babinet, M. F. d'Agay, J. P. Clauvel, M. Raphael, L. Degos, et al. 1995. Kaposi's sarcoma-associated herpesvirus-like DNA sequences in multicentric Castleman's disease. Blood 86:1276-1280.

33. Sun, R., S. F. Lin, L. Gradoville, Y. Yuan, F. Zhu, and G. Miller. 1998. A viral gene that activates lytic cycle expression of Kaposi's sarcoma-associated herpesvirus. Proc. Natl. Acad. Sci. USA 95:10866-10871.

34. Sun, R., S. F. Lin, K. Staskus, L. Gradoville, E. Grogan, A. Haase, and G. Miller. 1999. Kinetics of Kaposi's sarcoma-associated herpesvirus gene expression. J. Virol. 73:2232-2242.

35. Verma, S. C., and E. S. Robertson. 2003. Molecular biology and pathogenesis of Kaposi sarcoma-associated herpesvirus. FEMS Microbiol. Lett. 222:155163.

36. Wang, Y., O. T. Chong, and Y. Yuan. 2004. Differential regulation of K8 gene expression in immediate-early and delayed-early stages of Kaposi's sarcomaassociated herpesvirus. Virology 325:149-163.

37. West, J. T., and C. Wood. 2003. The role of Kaposi's sarcoma-associated herpesvirus/human herpesvirus-8 regulator of transcription activation (RTA) in control of gene expression. Oncogene 22:5150-5163.

38. Whitby, D., V. A. Marshall, R. K. Bagni, C. D. Wang, C. J. Gamache, J. R. Guzman, M. Kron, P. Ebbesen, and R. J. Biggar. 2004. Genotypic characterization of Kaposi's sarcoma-associated herpesvirus in asymptomatic infected subjects from isolated populations. J. Gen. Virol. 85:155-163.

39. Zhu, F. X., T. Cusano, and Y. Yuan. 1999. Identification of the immediateearly transcripts of Kaposi's sarcoma-associated herpesvirus. J. Virol. 73: 5556-5567.

40. Zong, J. C., C. Metroka, M. S. Reitz, J. Nicholas, and G. S. Hayward. 1997. Strain variability among Kaposi sarcoma-associated herpesvirus (human herpesvirus 8) genomes: evidence that a large cohort of United States AIDS patients may have been infected by a single common isolate. J. Virol. 71: 2505-2511. 